\title{
The remarkable blue-shift dominated jet in the high mass protostellar object G353.273+0.641
}

\section{Kazuhito Motogi*广}

The Research Institute for Time Studies, Yamaguchi University

E-mail: motogi@yamaguchi-u.ac.jp

\section{Kazuo Sorai}

Department of Physics, Faculty of Science, Hokkaido University

E-mail: sorai@astrol.sci.hokudai.ac.jp

\section{Kenta Fujisawa}

The Research Institute for Time Studies, Yamaguchi University

E-mail: kenta@yamaguchi-u.ac.jp

\section{Koichiro Sugiyama}

Department of Physics, Faculty of Science, Yamaguchi University

E-mail: koichiro@yamaguchi-u.ac.jp

\section{Kotaro Niinuma}

Department of Physics, Faculty of Science, Yamaguchi University

E-mail: kotaro@yamauchi-u.ac.jp

\section{Mareki Honma}

MizusawaVLBI Observatory, National Astronomical Observatory of Japan

E-mail: mareki.honmalnao.ac.jp

The dominant blue-shifted maser (DBSM) is a $22 \mathrm{GHz}$ water maser source that shows a significantly asymmetric spectrum, where almost all flux is concentrated in blue-shifted emission with respect to the systemic velocity. It is a candidate of high mass protostellar object with a pole-on jet. We have found two thermal counterparts of the archetypal DBSM, G353.273+0.641, (1) an extended radio jet, (2) an extremely high-velocity and unusually broad $\mathrm{SiO}(\mathrm{v}=0, \mathrm{~J}=2-1)$ jet. The radio jet is extended along NW-SE direction and the masers are located at the root part of the jet. On the other hand, spectral features of $\mathrm{SiO}$ jet are very similar to that of the maser, i.e., it is clearly blue-shift dominated. This strongly suggests that this $\mathrm{SiO}$ jet is directly related to the maser excitation. If this is the case, the scale of the $\mathrm{SiO}$ jet can be comparable with the maser distribution. A clear bipolar structure of the radio jet suggests that the observed blue-shift dominance cannot be explained by a simple asymmetric jet. Alternatively, it can arise from a masking of a red-shifted side by an optically thick disk of 100 milli-arcsecond scale.

11th European VLBI Network Symposium \& Users Meeting,

October 9-12, 2012

Bordeaux, France

\footnotetext{
* Speaker.

$\dagger$ This work was financially supported by the Grant-in-Aid for the JSPS Fellows (KM).
} 


\section{Introduction: the dominant blue-shifted maser G353.273+0.641}

The dominant blue-shifted maser (DBSM) is a class of $22 \mathrm{GHz} \mathrm{H}_{2} \mathrm{O}$ maser sources that show a significantly asymmetric spectrum, i.e., almost all flux is concentrated in blue-shifted emission with respect to the systemic velocity ([1]; hereafter CP08). It is thought to be a candidate of high mass protostellar object (HMPO) with a pole-on protostellar jet, because of its broad velocity range (up to $\pm 100 \mathrm{~km} \mathrm{~s}^{-1}$ ) [1, 2]. A statistical study of $\mathrm{CH}_{3} \mathrm{OH}$ masers associated with $\mathrm{H}_{2} \mathrm{O}$ masers, but without $\mathrm{OH}$ masers (i.e., the youngest population) in Breen et al. (2010) [3] shows that the number of blue-shift dominated sources is clearly larger than the red-shift dominated sources. Such a statistical anomaly requires some intrinsic mechanism to cause strong blue-shift dominance.

The target source, G353.273+0.641 (hereafter G353), is the initially-identified and archetypal DBSM at a distance of $1.7 \mathrm{kpc}$ [1]. Figure 1 shows the interferometric spectrum of the $\mathrm{H}_{2} \mathrm{O}$ maser in G353, where the total velocity range reaches $210 \mathrm{~km} \mathrm{~s}^{-1}$ and the peak emission is significantly blue-shifted $\left(\sim-50 \mathrm{~km} \mathrm{~s}^{-1}\right)$ with respect to the systemic velocity of $-5 \mathrm{~km} \mathrm{~s}^{-1}$. Motogi et al. (2011) ([2]; hereafter MO11) have reported our VLBI and single-dish monitoring study towards G353. Figure 2 presents the overall distribution of $\mathrm{H}_{2} \mathrm{O}$ maser spots detected during the initial 2-yr monitoring with the VLBI Exploration of Radio Astrometry (VERA) [2]. All maser spots spread over the extremely wide velocity range $\left(\sim 100 \mathrm{~km} \mathrm{~s}^{-1}\right)$ are clustered in a very compact region of about $\sim 200 \times 100$ milli-arcsecond ${ }^{2}\left(\right.$ mas $\left.^{2}\right)$.

One intriguing characteristic of G353 is the significant variability of the $\mathrm{H}_{2} \mathrm{O}$ maser. The maser light curve during four years is presented in Figure 2, and one can see highly intermittent maser flare activities. MO11 have also found that these flares are accompanied by systematic change of spatial distribution and synchronized acceleration of maser clusters, suggesting a recurrent shock propagation. MO11 have concluded that such a recurrent shock can be caused by a precessing or episodic protostellar jet.

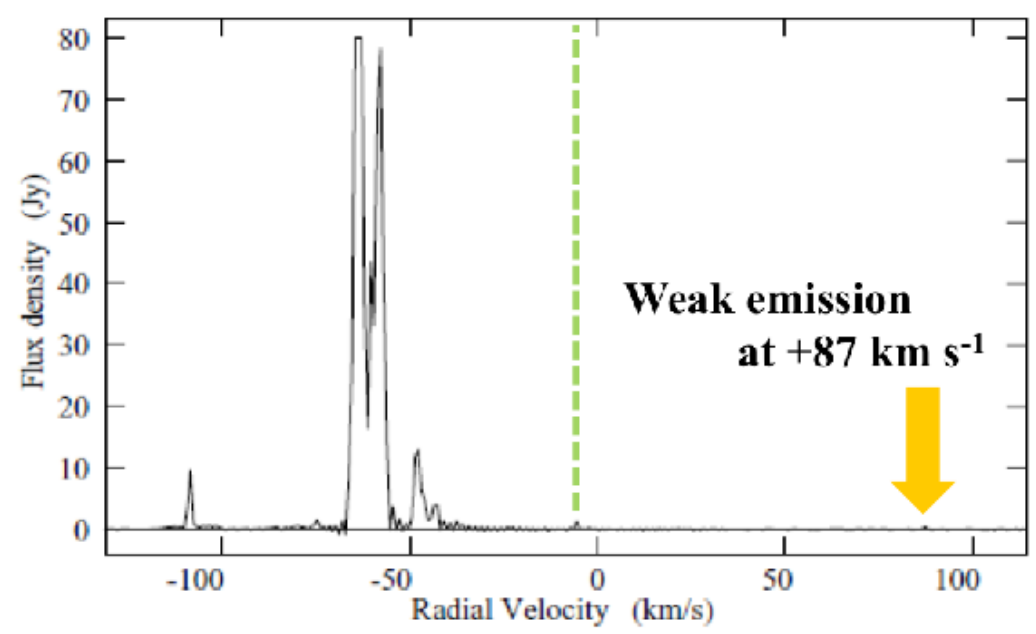

Figure 1: An interferometric spectrum of the $\mathrm{H}_{2} \mathrm{O}$ maser in $\mathrm{G} 353$ taken by the Australia Telescope Compact Array (ATCA) from CP08. The systemic velocity of $-5 \mathrm{~km} \mathrm{~s}^{-1}$, which is estimated from the associated class II $\mathrm{CH}_{3} \mathrm{OH}$ maser emission at $6.7 \mathrm{GHz}$, is indicated by the green dotted line. The strongest peak of $240 \mathrm{Jy}$ is truncated at $80 \mathrm{Jy}$, in order to emphasize relatively weak components (see CP08 for more details) 

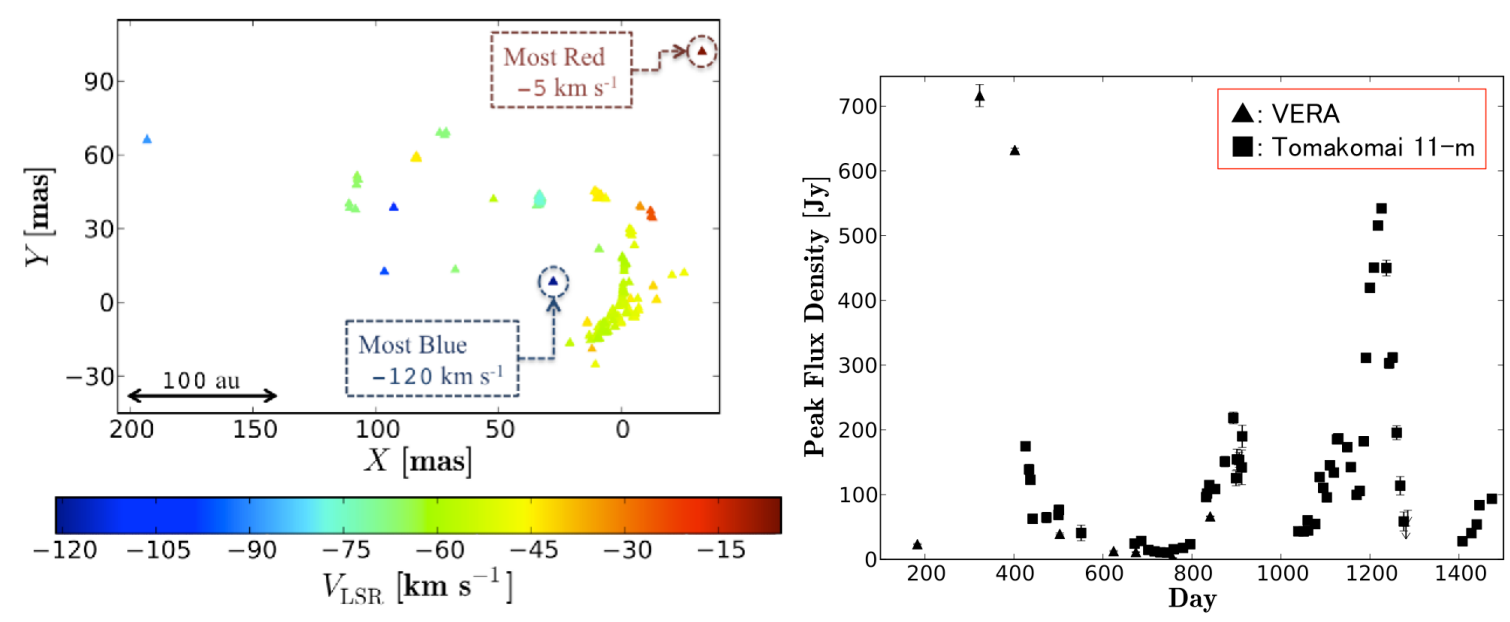

Figure 2: Left: Spatial distribution of $\mathrm{H}_{2} \mathrm{O}$ maser spots in $\mathrm{G} 353$ from MO11. The coordinate origin is $\alpha_{2000}=17^{h} 26^{m} 01^{s} .5883, \delta_{2000}=-34^{\circ} 15^{\prime} 14^{\prime \prime} .905$. Each triangle indicates a detected maser spot and its color represents a line-of-sight (LOS) velocity. It should be noted that the extremely red-shifted component at $+87 \mathrm{~km} \mathrm{~s}^{-1}$ reported in CP08 (see Figure 1) is out of the observing bandwidth in MO11, and the lowest velocity component is consistent with the systemic velocity [1]. Right: The light curve of the main velocity component of the $\mathrm{H} 2 \mathrm{O}$ maser $\left(-53 \pm 7 \mathrm{~km} \mathrm{~s}^{-1}\right.$; see MO11 in details). The $x$-axis indicates relative day from 2008 January 1. Filled squares and triangles corresponds to data taken with the VERA and the Hokkaido University Tomakomai 11-m radio telescope, respectively.

\section{Follow-up observations of a high mass protostellar jet}

Since there was no direct observation of a jet itself, we conducted a radio and molecular jet survey in order to reveal the relation between a jet and $\mathrm{H}_{2} \mathrm{O}$ masers. Results have already been published in Motogi et al. (2013) [4], and here, we focus only on scientific results (see Motogi et al. 2013 for additional details).

A radio jet survey was performed using the ATCA. Figure 3 presents the image of the detected radio jet with the positions of all the maser spots detected in MO11. This is the first detection of a host protostellar jet associated with a known DBSM, supporting a previous pole-on jet hypothesis in $\mathrm{CP} 08$ and MO11. The spectral index estimated from the total flux of 18 and $22 \mathrm{GHz}$ continuum is $2.6 \pm 1.0$, indicating an optically thick free-free emission that is typical for a radio jet from HMPOs (e.g., Hofner et al. 2007 [5]). $\mathrm{H}_{2} \mathrm{O}$ masers clearly trace the root of the bipolar jet, suggesting the presence of a molecular component of the jet near the driving source.

Such a molecular component has been detected with the $\mathrm{SiO}(v=0, J=2-1)$ line using the Nobeyama Radio Observatory 45-m telescope. Figure 3 also shows the spectrum of the detected extremely high-velocity $\mathrm{SiO}$ jet. The observed spectral features are very similar to that of the $\mathrm{H}_{2} \mathrm{O}$ maser, i.e., an extremely broad velocity range on the blue-shifted side $\left(-120--5 \mathrm{~km} \mathrm{~s}^{-1}\right)$, no emission on the red shifted side $\left(0-80 \mathrm{~km} \mathrm{~s}^{-1}\right)$ and an isolated emission at $+87 \mathrm{~km} \mathrm{~s}^{-1}$ (see also Figure 1). This spectral similarity strongly suggests that the $\mathrm{SiO}$ jet is directly related to the maser excitation, i.e., it probably traces strong shocks at the root of the bipolar jet. If this is the case, the scale of the $\mathrm{SiO}$ jet can be comparable with the maser distribution, i.e., only 200 mas ( $340 \mathrm{au})$. 

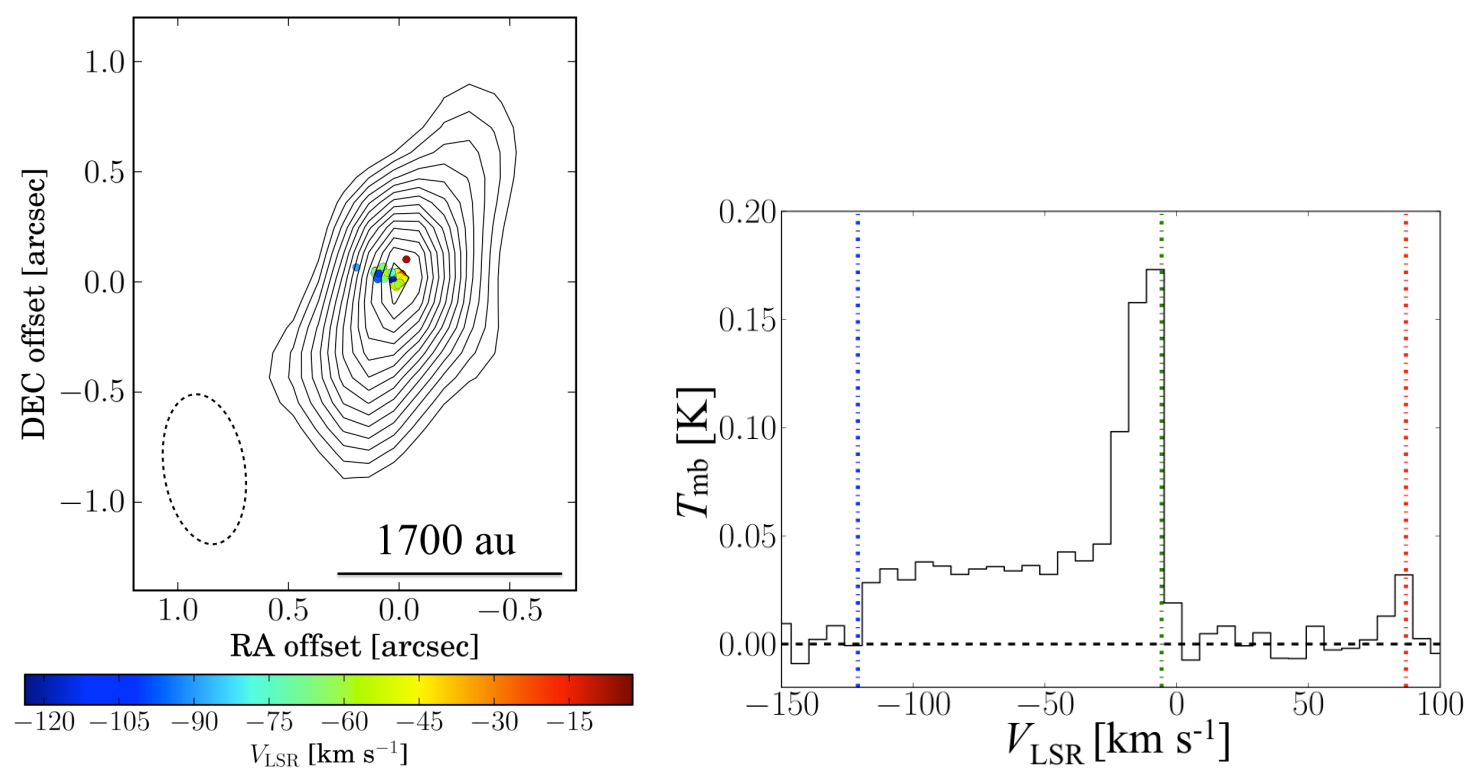

Figure 3: Left: the contour image of the radio jet in $\mathrm{G} 353$ at $22 \mathrm{GHz}$. The coordinate origin is same as on the maser map in Figure 2. The contour levels are in steps of $37 \mu \mathrm{Jy}_{\text {beam }}{ }^{-1}$ and the first contour level starts from 3- $\sigma\left(111 \mu \mathrm{Jy}_{\text {beam }}{ }^{-1}\right)$. A synthesized beam of $0^{\prime \prime} .69 \times 0^{\prime \prime} .36$ is shown in the lower left corner. Color points show $\mathrm{H}_{2} \mathrm{O}$ maser spots with LOS velocities (see also Figure 2). Right: the $\mathrm{SiO}(v=0, J=2-1$ ) spectrum in the main beam brightness temperature, $T_{\mathrm{mb}}$, scale. The blue and red dotted lines indicate the most red $\left(+87 \mathrm{~km} \mathrm{~s}^{-1}\right)$ and blue-shifted $\left(-120 \mathrm{~km} \mathrm{~s}^{-1}\right)$ maser component, respectively (see Figure 1). The systemic velocity of $-5 \mathrm{~km} \mathrm{~s}^{-1}$ is also indicated by the green dotted line. The black horizontal line shows the zero level.

\section{Discussion: possible origin of the blue-shift dominance}

The detection of the remarkably blue-shift dominated $\mathrm{SiO}$ jet manifests that the fundamental origin of DBSMs is not a matter of the radiative transfer of $\mathrm{H}_{2} \mathrm{O}$ masers that was discussed in CP08. The most simple explanation for the observed blue-shift dominance in the maser and the $\mathrm{SiO}$ emission is a highly asymmetric jet structure. However, this is an unlikely case, since the radio jet shows a clear bipolar structure. Also, this cannot explain the statistical anomaly mentioned above. Another possible explanation is that the red-shifted emission is masked by a disk which is optically thick at 22 and $86 \mathrm{GHz}$. Observational signatures of such an optically thick disk are reported for several sources (e.g., [6, 7]).

Figure 4 shows a schematic view of the expected source geometry. This scenario can consistently explain why DBSMs are observed in the youngest evolutional phase of HMPOs [3], i.e., a younger object should have a more massive and dense accretion disk. Expected pole-on geometry of DBSMs is also suitable for the scenario, for example, the inclination angle of the jet in G353 estimated from the LOS velocity and the maser proper motions is smaller than $30^{\circ}$. This pole-on geometry can allow a disk to mask a significant fraction of the red-shifted jet, although the detection of the extremely red-shifted component of $+87 \mathrm{~km} \mathrm{~s}^{-1}$ suggests that the masking effect is incomplete, possibly because of a relatively small disk size compared to the jet ( $\sim 100$ mas).

If our scenario is true, the optically thick $22 \mathrm{GHz}$ continuum emission detected by the ATCA 


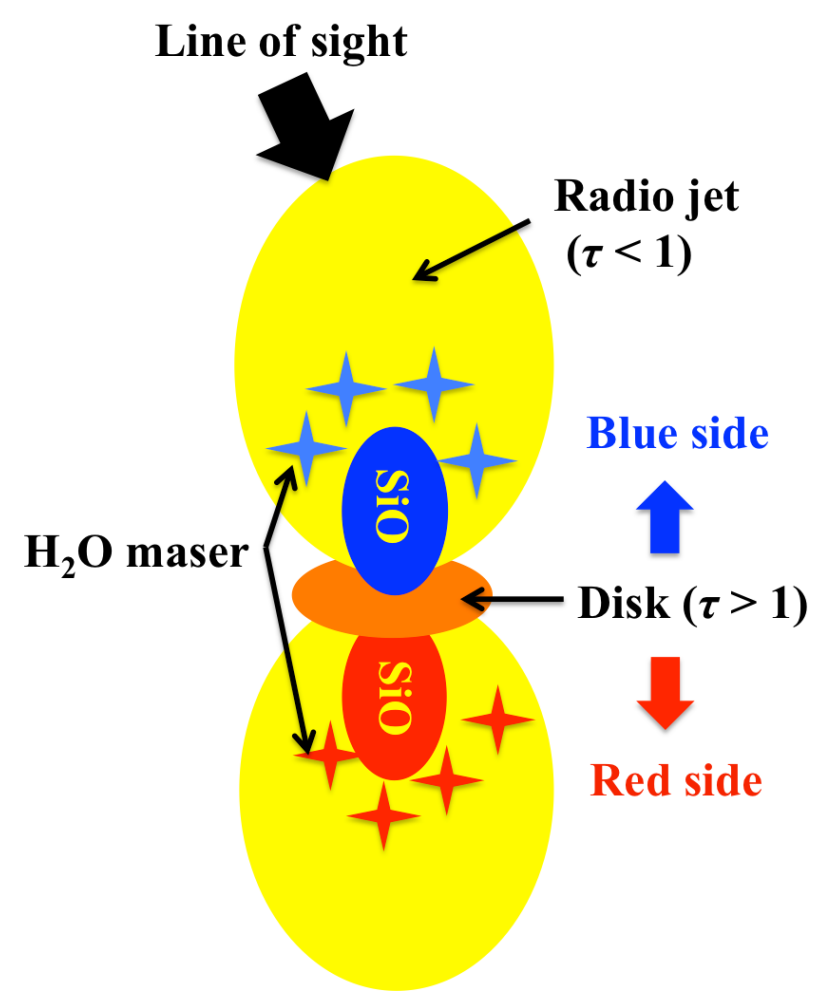

Figure 4: Schematic view of the source geometry in our disk-masking scenario.

should be a combination of an unresolved optically thick disk and an extended radio jet. The apparent geometry, where blue-shifted masers are enclosed by the radio jet, may require that the radio jet itself is optically thin at $22 \mathrm{GHz}$. A direct imaging of the unusual $\mathrm{SiO}$ jet and $\mathrm{cm}-\mathrm{mm}$ continuum observations of G353 with a 100-mas resolution is absolutely necessary to verify our scenario. It will be a first step of further statistical discussion about the exact nature of DBSMs.

\section{References}

[1] Caswell J. L., Phillips C. J., 2008, MNRAS, 386, 1521 (CP08)

[2] Motogi K., et al., 2011, MNRAS, 417, 238 (MO11)

[3] Breen S. L., Caswell J. L., Ellingsen S. P., Phillips C. J., 2010, MNRAS, 406, 1487

[4] Motogi K., et al., 2013, MNRAS, 428, 349

[5] Hofner P., Cesaroni R., Olmi L., Rodríguez L. F., Martí J., Araya E., 2007, A\&A, 465, 197

[6] Beuther H. et al., 2004, ApJ, 616, L31

[7] Hirano N., Ho P. T. P., Liu S.-Y., Shang H., Lee C.-F., Bourke T. L., 2010, ApJ, 717, 58 